\title{
Evaluation of the Transportation Resources Allocation Efficiency Based on the Production Frontiers
}

\author{
Rongyu Wu \\ School of Economics and Management \\ Beijing Jiaotong University \\ Beijing, China \\ 12120563@mail.bjtu.edu.cn
}

\author{
Hongchang Li \\ School of Economics and Management \\ Beijing Jiaotong University \\ Beijing, China \\ hchli@mail.bjtu.edu.cn
}

\begin{abstract}
Reasonable allocation of transportation resources is an important support and guarantee of the development of transportation. And the efficiency of transportation resources allocation is an important basis of transportation resources distribution. We could analysis the distribution of transportation resources from two aspects, which are scale and structure. According to the theoretical analysis, we put forward the evaluation methods and models of transportation resources allocation efficiency on the basis of production frontiers theory. This paper verified the established evaluation and optimization theory of the transportation resources allocation efficiency is feasible and effective according to the empirical research during 1992 2011.
\end{abstract}

Keywords-transportation; transportation resources; allocation efficiency; production frontiers

\section{THE THEORETICAL BASIS FOR TRANSPORTATION RESOURCES ALLOCATION EFFICIENCY}

\section{A. Efficiency of Transportation Resources Allocation}

The original concept of efficiency is "useful work per quantity of energy". Efficiency is the comparison of the two kinds of similar things. The efficiency of transportation resources allocation is the ratio of actual configuration and ideal configuration level of transportation resources. The efficiency of transportation resources allocation is a concept of general efficiency, which reflects the degree of resource utilization. It can be decomposed into scale efficiency and structure efficiency of comprehensive transportation network.

The scale efficiency of transportation comprehensive network (SE) is the level of valid state compared with economies of scale in transportation network under the condition of transportation technology unchanged. [1] The fundamental reasons for the existence of scale efficiency of transportation comprehensive network are the complementary of technical and economic characteristics of various modes of transportation, moreover, transport resources or elements are non-severability. When combination of various modes of transportation is fixed and transportation technology is constant, the scale efficiency of transportation comprehensive network (SE) can be represented as the ratio of transportation output per unit cost $(Y / C)$ and when it come to economies of scale $\left(Y_{S} / C_{S}\right)$, or the ratio of unit transportation cost of products at economies of scale $\left(C_{S} / Y_{S}\right)$ and real unit transportation cost of products $(C / Y)$.

$$
S E=\frac{Y / C}{Y_{S} / C_{S}}=\frac{C_{S} / Y_{S}}{C / Y}
$$

The structural efficiency of transportation comprehensive network (STE) is the level of valid state compared with the optimal degree of transportation network structure when the scale of transport resource investment unchanged. The structural efficiency transportation comprehensive network (STE) can be represented as the ratio of real output $(Y)$ and maximum output $\left(Y_{C}\right)$ when the scale is constant and structure is optimal, or the ratio of minimum cost $\left(C_{C}\right)$ when complete equal transportation products at the same scale and the real cost $(C)$, there is consistency between the two expression

$$
T E=\frac{Y}{Y_{T}}=\frac{C_{T}}{C}
$$

The efficiency of transportation resources allocation $(E)$ can be obtained by the product of the scale efficiency of transportation comprehensive network $(S E)$ and the structural efficiency of transportation comprehensive network $(C E)$.

$$
E=S E \times C E
$$

The value of scale efficiency and structural efficiency ranges from 0 and 1 , and thus the value of the efficiency of transportation resources allocation $(E)$ also ranges from 0 and 1. When the value of scale efficiency is $1,(S E=1)$, the value of construct efficiency is $1,(C E=1)$, and the value of the efficiency of transportation resources allocation is also 1 , $(E=1)$, it is called completely scale efficiency, completely structural efficiency and completely allocation efficiency. Only when all these efficiency reached 1, the efficiency of transportation resources allocation achieve the optimal.

\section{B. The Economic Sense of Transportation Resources Allocation}

We apply isocost line of equal transportation volume to illustrate the economic sense of transportation resources allocation, as shown in the figure 1. To facilitate the analysis, assume that there two kinds of transportation production 
resources, $X_{1}$ and $X_{2}$. At the real production point $K$, the output is $Y$, the cost is a, $A A$ represent isocost line of equal transportation volume. $O P$ is ratio line of transportation resources combination, which means all the points on $O P$ have the same combined ratio. It should be noted that the curves AA, BB, CC represent different cost level of same output $Y$. The isocost line $R R$ and the isocost line of equal transportation volume $C C$ tangent at $d$.

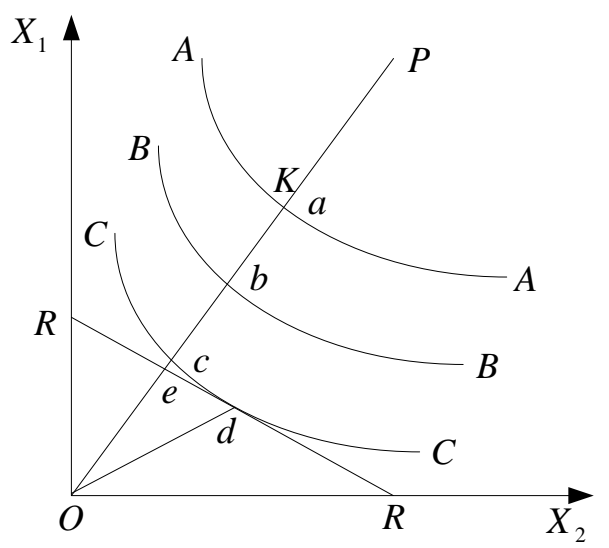

Figure 1. Isocost line of equal transportation volume

We discuss in more detail the influence of SE and STE.

First is about SE, when the structure of transportation remains the same, the economies of scale could be achieved by change the input of transportation resources. Assume that transportation volume $Y$ is constant, isocost line move from $A A$ to $B B$, the input cost move from $a$ to $b$, which is ideal cost point of scale efficiency, and implement the goal of saving transportation resources $X_{1}$ and $X_{2}$. Now the $\mathrm{SE}$ at production point $K$ can be written as follows

$$
S E_{K}=\frac{O b}{O a}
$$

where $a b$ is the amount of non-scale efficiency.

Second is about STE, there is mutual cooperation of various modes of transportation, and the scale of input remains the same. Isocost line move from $B B$ to $C C$, the input cost move from $b$ to $c$, meanwhile, the reasonable allocation of transportation structure can make transportation resources to achieve the optimal combination. Point $d$ is the minimum cost point which is determined by the price of transportation resources. And thus $O d$ is the ratio line of transportation resources combination when the cost is minimum, thus also implement the goal of saving transportation resources $X_{1}$ and $X_{2}$. Therefore, input cost $c$ will move further to $d$, which represent the ideal cost of structure efficiency. Assume that $R R$ and $O P$ tangent at $e$, now the STE at production point $K$ can be written as follows

$$
C E_{K}=\frac{O d}{O b}=\frac{O e}{O b}
$$

where $e b$ is the amount of non-structure efficiency.
Therefore the efficiency of transportation resources allocation of point $K$ is

$$
E_{K}=S E_{K} \times T E_{K}=\frac{O b}{O a} \times \frac{O e}{O b}=\frac{O e}{O a}
$$

where $a e$ is the amount of non efficiency.

The moving of cost from $a$ to $e$ indicates that the enhance of scale efficiency and structure efficiency make the allocation of transportation resources investment more efficient. Implement the goal of saving transportation resources and reduction of costs, without reduce the output. We can also inferred that the key to evaluate the efficiency of transportation resources allocation is econometric estimation of scale efficiency frontiers $B B$ and structure efficiency frontiers $C C$.

\section{THE DEFINITION OF THE TRANSPORTATION PRODUCTION FRONTIERS}

Assume that the input of transportation production is $X=\left(x_{1}, x_{2}, \cdots, x_{m}\right)^{T}, \quad X \in E^{m}, E^{m}$ is a combination set of input factor. The output of transportation production is $Y=\left(y_{1}, y_{2}, \cdots, y_{n}\right)^{T}, \quad Y \in E^{n}, E^{n}$ is a combination set of output factor. Thus the production possibility set is technically combination between the feasible input and potential output $(X, Y),(X, Y) \in T$, that is a collection of all possible transportation production activities. It gives a complete description of the input-output possibility of production system under the current technical conditions.

$$
T=\left\{(X, Y) \mid X>0, Y>0, X \in E^{m}, Y \in E^{m}\right\}
$$

In the collection of all possible transportation production, the combination of effective transportation resources input and the maximum possible output $(\bar{X}, \bar{Y}) \in T$ draws great attention, all the collection of production points $(\bar{X}, \bar{Y})$ called the production frontiers of production possible collection $T$. We can use function to indicate the composition relationship between input $\bar{X}$ and output $\bar{Y}$ of transportation production frontiers.

$$
\bar{Y}=f(\bar{X})
$$

That is to say, production frontiers function is a mathematical expression of dependence relationship between the combination of transportation resources input and maximum transportation volume or traffic turnover. For all the production points $(\bar{X}, \bar{Y})$, there is $Y \leq \bar{Y}$. The curve of production frontiers function is the external boundary of all possible combinations of input-output, all the observations of input and output coordinates located below this boundary and as close as possible to it. When the production point fall on the curve of production frontiers function, it means the corresponding input and output status is the best technical level, and the structural efficiency of transportation resources allocation is optimal. When the production point fall below the 
curve of production frontiers function, it means the usage of input resources does not meet the best technical level, the difference between the frontiers and the actual production level is due to the loss caused by scale efficiency and structural efficiency did not reach the optimal. [2]

In the measurement of efficiency of transportation resources allocation, the research of production frontiers has the extremely vital significance. This is because the real transportation input-output status could be obtained through the actual statistical data, but the ideal resource allocation situation is difficult to draw. Production frontiers (function) express an ideal resource allocation situation. The research of production frontiers will contribute to the measurement of efficiency of transportation resources allocation. There are basically two kinds of methods to measure production frontiers, parametric method and non-parametric method.

The parametric method is assuming the input and output of production activity correspond to some functional form, and then estimate the parameters through the appropriate statistical methods, thus result production function.

Assume the number of transportation production decision making unit is $n$, each decision making unit, $D M U_{j}(j=1,2 \cdots, n)$, has $m$ kinds of transportation resources input and $s$ kinds of transportation resources output, input $X_{j}=\left(x_{1 j}, x_{2 j}, \cdots, x_{m j}\right)^{T},(j=1,2 \cdots, n)$ represent unit cost of transportation resources, and output $Y_{j}=\left(y_{1 j}, y_{2 j}, \cdots, y_{s j}\right)$, $(j=1,2 \cdots, n)$ represent transportation volume. As shown in figure 2 .

\begin{tabular}{|c|c|c|c|c|c|}
\hline & $D M U_{1}$ & $D M U_{2}$ & $\cdots$ & $D M U_{n}$ & \\
\hline$v_{1} \quad 1 \rightarrow$ & $x_{11}$ & $x_{12}$ & $\cdots$ & $x_{1 n}$ & \\
\hline$v_{2} 2 \rightarrow$ & $x_{21}$ & $x_{22}$ & $\cdots$ & $x_{2 n}$ & \\
\hline$\vdots \quad \vdots \rightarrow$ & $\vdots$ & $\vdots$ & $\vdots$ & $\vdots$ & \\
\hline \multirow[t]{5}{*}{$v_{m} m \rightarrow$} & $x_{m 1}$ & $x_{m 2}$ & $\cdots$ & $x_{m n}$ & \\
\hline & $y_{11}$ & $y_{12}$ & $\cdots$ & $y_{1 n}$ & $\rightarrow 1 \quad u_{1}$ \\
\hline & $y_{21}$ & $y_{22}$ & $\cdots$ & $y_{2 n}$ & $\rightarrow 2 \quad u_{2}$ \\
\hline & $\vdots$ & $\vdots$ & $\vdots$ & $\vdots$ & $\rightarrow \vdots \quad \vdots$ \\
\hline & $y_{s 1}$ & $y_{s 2}$ & $\cdots$ & $y_{s n}$ & $\rightarrow s \quad u_{s}$ \\
\hline
\end{tabular}

Figure 2. The definition of transportation decision making unit

Where means the input in the $i$ th type of the $j$ th DMU, means the output in the $r$ th type of the $j$ th DMU, is weight which measure the $i$ th type of input, measures the $r$ th type of output, and $x_{i j}>0, y_{r j}>0, v_{i} \geq 0, u_{r} \geq 0$, $i=1,2, \cdots, m, i=1,2 \cdots, m, r=1,2 \cdots, s \quad, \quad j=1,2 \cdots, n$. $\left(x_{i j}\right.$ and $y_{r j}$ are given data based on historical data and prediction)

Corresponding to the weight $V=\left(v_{1}, v_{2}, \cdots, v_{m}\right)^{T}$, $U=\left(u_{1}, u_{2}, \cdots, u_{s}\right)^{T}$, we define evaluation index of each decision making unit $H_{j}$

$$
H_{j}=\frac{U^{T} Y_{j}}{V^{T} X_{j}}, \quad j=1,2 \cdots, n
$$

For Eq. (9), the weight $V$ and $U$ could be chose properly to satisfy $H_{j} \leq 1, \quad j=1,2 \cdots, n$.

Then we evaluate the efficiency of $D M U_{j_{0}}, 1 \leq j_{0} \leq n$. Choose the weight $V$ and $U$ so $H_{j}$ could reach the maximum under the condition that each evaluation index of $D M U$ is no more than 1 . Thus the optimal model is as follows

$$
(\bar{P})\left\{\begin{array}{lc}
\max & H_{j 0}=\frac{U^{T} Y_{j 0}}{V^{T} X_{j 0}}=V_{\bar{P}} \\
\text { s.t. } & H_{j}=\frac{U^{T} Y_{j}}{V^{T} X_{j}} \leq 1, j=1,2, \cdots, n \\
& V \geq 0, U \geq 0
\end{array}\right.
$$

$(\bar{P})$ is a fractional programming model, it can be turned into a linear programming problem by equivalent transformation of Charnes-Cooper exchange.

$$
(P) \begin{cases}\max & \mu^{T} Y_{j 0}=V_{P} \\ \text { s.t. } & \omega^{T} X_{j}-\mu^{T} Y_{j} \geq 0, j=1,2, \cdots, n \\ & \omega^{T} X_{j 0}=1 \\ & \omega \geq 0, \mu \geq 0\end{cases}
$$

Introduce slack variables $S^{+}$and $S^{-}$, the dual program of linear program $(P)$ is

$$
(D) \begin{cases}\min & \theta=V_{D} \\ \text { s.t. } & \sum_{j=1}^{n} X_{j} \lambda_{j}+S^{-}=\theta X_{j 0} \\ & \sum_{j=1}^{n} Y_{j} \lambda_{j}-S^{+}=Y_{j 0} \\ & \lambda_{j} \geq 0, j=1,2, \cdots, n ; S^{+} \geq 0, S^{-} \geq 0\end{cases}
$$

The function outlined above is $C^{2} R$ model, the definition of $C^{2} R$ model under $D E A$ validity is if there are optimal solutions $w_{j_{0}}$ and $\mu_{j_{0}}$, which satisfy $V_{P}=\mu_{j 0}{ }^{T} Y_{j 0}=1$ and $\omega_{j 0}>0, \mu_{j 0}>0, D M U_{j 0}$ is $D E A$ validity.

By the linear duality theory and "elastic" quantitative, we know that for $D M U_{j 0}$ is $D E A$ validity, the necessary and sufficient conditions is the optimal value of programming problem $(D)$ is $V_{D}=\theta_{j 0}=1$, and each optimal solution $\lambda_{j 0 i}=\left(\lambda_{j 01}, \lambda_{j 02}, \cdots, \lambda_{j 0 n}\right)^{T}$ satisfy $S_{j 0}^{+}=S_{j 0}^{-}=0$.

In practical application, we introduce the Archimedes dimensionless, transfer the programming (P) and (D) to $\left(P_{\tau}\right)$ 
and $\left(D_{\tau}\right)$, thus to facilitate the use of linear programming simplex method to solve the model.

$$
\begin{gathered}
\left(P_{\varepsilon}\right)\left\{\begin{array}{cc}
\max \quad \mu^{T} Y_{j 0}=V_{P \varepsilon} \\
\text { s.t. } \quad \omega^{T} X_{j}-\mu^{T} Y_{j} \geq 0, j=1,2, \cdots, n \\
& \omega^{T} X_{j 0}=1 \\
& \omega \geq \varepsilon e^{T}, \mu \geq \varepsilon \hat{e}^{T}
\end{array}\right. \\
\left(D_{\varepsilon}\right)\left\{\begin{array}{cc}
\min \quad\left[\theta-\varepsilon\left(\hat{e}^{T} S^{-}+\varepsilon e^{T} S^{+}\right)\right]=V_{D \varepsilon} \\
\text { s.t. } \quad \sum_{j=1}^{n} X_{j} \lambda_{j}+S^{-}=\theta X_{j 0} \\
\sum_{j=1}^{n} Y_{j} \lambda_{j}-S^{+}=Y_{j 0} \\
\lambda_{j} \geq 0, j=1,2, \cdots, n ; S^{+} \geq 0, S^{-} \geq 0
\end{array}\right.
\end{gathered}
$$

Where $\hat{e}^{T}=(1,1, \cdots, 1) \in E_{m} e^{T}=(1,1, \cdots, 1) \in E_{s}$.

The theorems for judging whether $D M U_{j 0}$ is $D E A$ validity by using $\left(D_{\varepsilon}\right)$ are: assume that is Archimedes dimensionless, and the optimal solution of programming problem $\left(D_{\varepsilon}\right)$ is $\lambda_{j 0}, S_{j 0}^{-}, S_{j 0}^{+}, \theta_{j 0}$. If $\theta_{j 0}=1, S_{j 0}^{-}=0, S_{j 0}^{+}=0$, then $D M U_{j 0}$ is $D E A$ validity.

If $D M U_{j 0}$ is $D E A$ validity, and the corresponding programming problem $\left(P_{\varepsilon}\right)$ has optimal solution $\omega_{j 0}$ and $\mu_{j 0}$, where $\omega_{j 0}>0, \mu_{j 0}>0, V_{P}=\mu_{j 0}^{T} Y_{j 0}=1$, and $\omega_{j 0}^{T} X_{j 0}=1$, thus $\omega_{j 0}^{T} X_{j 0}-\mu_{j 0}^{T} Y_{j 0}=0$, which means the point $\left(X_{j 0}, Y_{j 0}\right)$ located on the hyper-plane $\pi\left(\pi: \omega_{j 0}^{T} X_{j 0}-\mu_{j 0}^{T} Y_{j 0}=0\right)$. We could demonstrate that the DMUs represented by all the points on the hyper-plane $\pi$ are $D E A$ validity. The related content about $D E A$ model could be found in references [3-7].

\section{Evaluation Method AND Model}

Transportation resources allocation efficiency is the ratio of real allocation level $A$ and ideal allocation level $I$. The transportation resources allocation efficiency $E$ can be written as follows

$$
E=\frac{A}{I}=S E \times C E
$$

Transportation resources allocation efficiency reflects the degree of resource utilization. As previously mentioned, it can be decomposed into scale efficiency $(S E)$ and structure efficiency (STE) of comprehensive transportation network.

Microscopic transportation efficiency is operational efficiency of transportation enterprises. It reflects the efficiency of transportation resources allocation in transportation enterprises, which also include scale efficiency and structure efficiency. Although the scope of business, enterprise management mode, operation mode and management characteristics of each enterprises are different. But in essence, they must follow the same law. The general type of production frontiers function curve is shown in figure 3.

Obviously, production frontiers function is increasing function. Assume that $Y=f(X)$ is the production frontiers function of transportation resources input $X$ and transportation volume $Y$ in transportation enterprises. Based on the definition of frontier production function, the function express the maximum output $\mathrm{Y}$ when input is $X$, this is the ideal state of transportation resources input-output in transportation enterprises. Therefore, the points $(X, Y)$ represent the relationship of transportation resources input and output, all the points are ideal efficiency state (completely structure efficiency), such as $A, B$ in the figure.

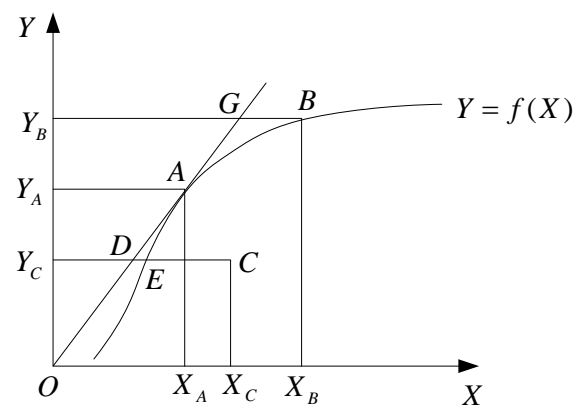

Figure 3. Transportation resources allocation efficiency

The function is divided into two parts by point $A$. In the left side of point $A$, the function accelerate, which means the increase of transportation resources input (expand the scale of operation) can increase transportation volume obviously, and marginal revenue is greater than marginal cost. This interval is called the stage of increasing return to scale. In the right side of point $A$, the curve becomes ramp up. The increase of transportation volume is slower than the increase of transportation resources input, and marginal revenue is smaller than marginal cost. This interval is called the stage of diminishing return to scale. Therefore, point $A$ represents the most appropriate input scale, where marginal revenue is equal to marginal cost, it achieve completely scale efficiency and completely technical efficiency. Point $B$ represents completely technical efficiency but not completely scale efficiency. Reduce the input of $B$ while maintaining its structural efficiency could increase unit capacity of traffic and improve the allocation efficiency. Point $\mathrm{C}$ is not on the curve of production frontiers function, the capacity input and output status it represented is obviously non-efficiency.

Scale efficiency (SE), structure efficiency (STE) and allocation efficiency $(\mathrm{E})$ of $\mathrm{A}, \mathrm{B}, \mathrm{C}$
A. $T E_{A}=1, S E_{A}=1, E_{A}=1$
B. $T E_{B}=1, S E_{B}=\frac{G Y_{B}}{B Y_{B}}<1, E_{B}=T E_{B} \times S E_{B}=\frac{G Y_{B}}{B Y_{B}}<1$
C. $T E_{C}=\frac{E Y_{C}}{C Y_{C}}<1, \quad S E_{C}=\frac{D Y_{C}}{E Y_{C}}<1, \quad E_{C}=T E_{C} \times S E_{C}=\frac{D Y_{C}}{C Y_{C}}<1$

Therefore, point $A$ represents completely efficiency of transportation resources allocation. Point $B$ represents completely structure efficiency, but the allocation efficiency is less than 1 because the scale efficiency is less than 1 , where $G B$ is the inefficient volume of supply. The scale efficiency 
and structure efficiency of point $C$ is less than 1 , thus allocation efficiency also less than 1 , where $C D$ is the inefficient volume of supply.

For the same mode of transportation, different transportation enterprises could make proper transportation combination and change independent production mode according to their own territory or the technical advantage, in order to gain more profit, such as readjusting the transportation structure. Assume that there is a transportation enterprise with completely allocation efficiency, then we analysis its production structure with many other transportation enterprises. In order to achieve completely efficiency, resources will be reallocated, which form the structure optimization process of same mode of transportation. The structure developed by the optimization process will lead to optimal resource allocation of this particular transportation industry. If the transportation resources allocation optimization expands in different modes of transportation all over the country, it will further lead to nationwide transportation structure optimization, and form efficient transportation network system nationwide. The implementation process of efficient transportation resources allocation structure can be expressed simply by figure 4 .

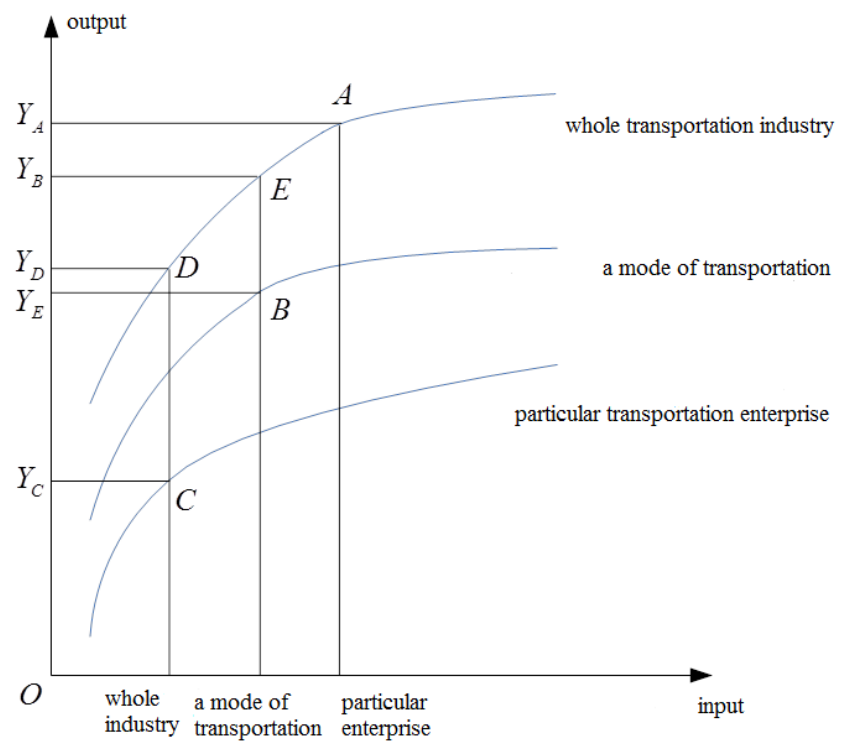

Figure 4. Transportation resources allocation

Point $C$ in figure 4 is completely allocation efficiency point of a particular transportation enterprise. Point $B$ is completely allocation efficiency point of multiple enterprises within the same mode of transportation. Point $A$ is completely allocation efficiency point of the whole transportation industry, thus readjust transportation structure and input according to point $A$ can achieve maximum profit. If with the best transportation structure as a reference object, the scale efficiency, structure efficiency and allocation efficiency of each point are

$$
\begin{array}{ll}
\text { Point } A & S E_{A}=1, \quad=1, E_{A}=1 \\
\text { Point } B & S E_{B}=\frac{Y_{E}}{Y_{A}}, S T E_{B}=\frac{Y_{B}}{Y_{E}}, E_{B}=\frac{Y_{B}}{Y_{A}}
\end{array}
$$

$$
\text { Point } C S E_{C}=\frac{Y_{D}}{Y_{A}}, S T E_{C}=\frac{Y_{C}}{Y_{D}}, E_{C}=\frac{Y_{C}}{Y_{A}}
$$

\section{EVALUATION AND ANALYSIS}

Under the support of transportation efficiency and optimization theory, this paper analyzed the transportation resources allocation efficiency of all the transportation modes in China. We base on data of 20 years (1992-2011), verified the evaluation and optimization theory of transportation resources allocation efficiency is feasible and effective.

\section{A. The Selection of Analysis Indicators}

\section{- Input of Transportation}

The input of transportation mainly includes three categories: capital, fuel and labor. They are represented by fixed investment of the year (including fixed assets update investment and new investment in fixed assets), conversion of the fuel consumption and number of employee.

\section{- $\quad$ Output of Transportation}

From the form of value, revenue is the indicator of transportation output. From the form of practical value, the indicator includes revenue passenger kilometers $(R P K)$ and freight ton-kilometers $(F T K)$. [11] In China, the fare policy is stable, and the transportation price is low and serious departure from the value of transportation. So it is unreasonable to use magnitude of value as indicator of output. Freight ton-kilometers is product of transportation volume and load distance which measured by ton-kilometers, it can correctly reflect the actual output of each transport mode. Therefore, we use $R P K$ and $F T K$ as the indicator of output.

Allow for the availability and accessibility of data, the number of employee, $R P K, F T K$ could be obtained from CHINA STATISTICAL YEARBOOK [8] and YEAR BOOK OF CHINA TRANSPORTATION and COMUNICATION. [9] Fixed investment could be obtained by the summary of fixed assets update investment and new investment in fixed assets. For data of fuel consumption, it is unable to get the data directly, so we get the data by different conversion methods according to the characteristics of the transportation industry.

\section{B. Estimation Results}

Table 1 expresses the results estimated by econometric software, and the result is 1 just mean its efficiency is relatively high rather than the absolute best of these $D M U s$. 
TABLE I.

TRANSPORTATION EFFICIENCY, 1992-2011

\begin{tabular}{|c|c|c|c|}
\hline & $S E$ & $S T E$ & $E$ \\
\hline 1992 & 0.4618 & 0.9990 & 0.4613 \\
\hline 1993 & 0.3739 & 0.9959 & 0.3724 \\
\hline 1994 & 0.3761 & 0.9580 & 0.3603 \\
\hline 1995 & 0.2779 & 0.9623 & 0.2674 \\
\hline 1996 & 0.0365 & 0.9858 & 0.0360 \\
\hline 1997 & 0.1051 & 0.9655 & 0.1015 \\
\hline 1998 & 0.4504 & 0.9543 & 0.4298 \\
\hline 1999 & 0.2349 & 0.9654 & 0.2268 \\
\hline 2000 & 0.5616 & 0.9766 & 0.5484 \\
\hline 2001 & 0.5656 & 0.9914 & 0.5607 \\
\hline 2002 & 0.5537 & 0.9989 & 0.5531 \\
\hline 2003 & 0.8562 & 1.0000 & 0.8562 \\
\hline 2004 & 1.0000 & 0.8687 & 0.8687 \\
\hline 2005 & 0.6296 & 0.9304 & 0.5858 \\
\hline 2006 & 0.5011 & 0.9574 & 0.4797 \\
\hline 2007 & 0.7099 & 0.9500 & 0.6744 \\
\hline 2008 & 0.4656 & 0.9704 & 0.4518 \\
\hline 2009 & 0.0448 & 0.9088 & 0.0407 \\
\hline 2010 & 0.8072 & 0.9437 & 0.7617 \\
\hline 2011 & 0.5585 & 0.9494 & 0.5302 \\
\hline
\end{tabular}

The results given above illustrate that the transportation resources allocation efficiency was low in China. This partly reflected the transportation development did not match with the national economic growth.

At the beginning of the reform and opening up, transportation development lagged behind the demand of national economy growth, thus the scale efficiency decreased year by year. It was widely acknowledged that the development of transportation will promote economic development. So the investment of transportation infrastructure had gradually increased. But due to hysteresis quality of the economic development caused by transportation development,[10] other issues have emerged, such as excessively rapid growth of transportation infrastructure and scale of construction, excess capacity, all these lead to the reduction of transportation resources allocation efficiency. ${ }^{[11]}$

With the rapid development of economy, the coordinated development of various modes of transportation has been realized. The market share of railway gradually declined, and highway, civil aviation gradually increased at the same time. But the input and output characteristics of different transportation modes is not the same, which railway belongs to low investment and high yield, highway and civil aviation belongs to high investment and low yield. But in recent years, energy conservation and emissions reduction had been taken seriously, the market share of railway increased correspondingly. At the same time as the change of the market share, structure efficiency also fluctuated.

The quantity and quality of transportation resources and their formation and service efficiency decides the scale and efficiency of transportation industry to a great extent, also determine the operative condition of the whole economic system.[12] Therefore, the evaluation of transportation resources allocation efficiency is of important significance for transportation industry structure and policy.

\section{REFERENCES}

[1] Hu Yin, "Efficiency analysis of transportation and economics of China based on fixed value chain," Science \& Technology Information, vol. 14 pp. 237-238, 2010.

[2] HAN Song, WEI Quan-ling, "The Nonparametric DEA Models for Resource Allocation," Systems Engineering - Theory \& Pratice, vol. 22, pp. 59-70, July 2002.

[3] Yu G, WeiQ L , B rocket t P, "A generalized data envelopment analysis model: a unification and extension of existing methods for efficiency analysis of decision making units," Annals of Operations Research, vol. 66, pp. 47-89, 1996.

[4] Charnes A, Cooper W W, Go lany B, Seiford L, Stutz J, "Foundations of data envelopment analysis for pareto-koop-mans efficient empirical product ion functions," Journal of Econometrics, vol. 30, pp. 91-107, 1985

[5] Charnes A, Cooper W W, WeiQ L, Huang ZM, "Cone ratio data envelopment analysis and multi-objective programming," International Journal of System Science, vol. 20, pp. 1099-1118, 1989.

[6] WeiQ L, Sun B D, Xiao Z J, "Measuring technical progress with data envelopment analysis," European Journal of Operational Research, vol. 80, pp.691-702, 1995.

[7] WeiQ L, DEA methods to Evaluate the Relative Effectiveness. Beijing, China Renmin University Press, 1987.

[8] National Bureau of Statistics of China, China Statistical Yearbook. Beijing, China Statistics Press, 1992-2012.

[9] China Transportation Statistics Press, Yearbook of China Transportation and Comunication, Beijing, China Transportation Statistics Press, 19922012.

[10] $\mathrm{Hu} \mathrm{Si}$-ji, Integrated Transportation Engineering, Beijing, Beijing Jiaotong University Press, 2005.

[11] Zhang Di, Wu Li-hua, "Empirical reaearch of the relationship between transportation infrastructure and economic growth in China," Journal of Industrial Technological Economics, vol. 27, pp. 87-90, August 2008.

[12] RONG Chao- he, "Necessity of economic analysis based on transport resources," Journal of Beijing Jiaotong University (Social Sciences edition ), vol. 5, pp. 1-7, December 2006. 